\title{
Hydration Sphere Structure of Proteins: a Theoretical Study
}

Anikó Lábas ${ }^{1}$, Imre Bakó ${ }^{2 *}$ and Julianna Oláh ${ }^{1 *}$

${ }^{1}$ Department of Inorganic and Analytical Chemistry, Budapest University of Technology and Economics, Szent Gellért tér 4, H-1111 Budapest, Hungary

${ }^{2}$ Institute of Organic Chemistry Research Centre for Natural Sciences, Hungarian Academy of Sciences Magyar tudósok körútja 2. H-1519 Budapest, P.O. Box 286, Hungary

.

*corresponding author, to whom corresponding should be addressed

e-mail: julianna.olah@mail.bme.hu; telephone number: +36-1-463-1286

e-mail: bako.imre@ttk.mta.hu; telephone number: +36-1-382-69-81 


\section{Abstract}

11 Hydration is essential for the proper biological activity of biomolecules. We studied the water 12 network around insulin (as a model protein) in aqueous $\mathrm{NaCl}$ solutions using molecular dynamics 13 simulations and statistical analysis of the topological properties (hydrogen bond neighbor number 14 and the interaction energy between hydrogen-bonded water molecules) of the water network. We 15 propose a simple method to define the hydration layers around proteins. Water molecules in the first 16 and second layers form significantly less, but stronger hydrogen bonds with each other than in the 17 bulk phase. Furthermore, water molecules over the hydrophilic and hydrophobic surface of the 18 protein possess slightly different H-bonding properties, supporting the hypothesis of structural and 19 dynamical heterogeneity of the water molecules over protein surface. The protein molecule perturbs 20 the solvent structure at least up to the fourth-fifth hydration layer. Our data suggest the peculiar role 21 of the second hydration shell.

25 Keywords: hydrogen-bond network, hydration sphere, MD simulations 
27 The appropriate spatial structure is essential for the activity of proteins. It is affected by both intramolecular interactions in the proteins and the intermolecular interactions formed with the solvent molecules, which is water in living cells. It has been well-established that the dominant conformational motions of proteins are profoundly affected by their hydration shell.[1,2] As a consequence, structural changes of the solvent should inevitably affect protein structure and function as well. Indeed, addition of compounds such as inorganic salts, organic molecules, acids or bases to the solution can perturb the structure of liquid water leading to the denaturation of the biomolecule. Among these, the denaturing effect of salts has been the most extensively studied, and more than a century ago Franz Hofmeister ordered the ions according to their ability to precipitate egg-white proteins. $[3,4]$ Kosmotropic ions (e.g. sulfate ion) or water structure makers strengthen the hydrogenbonding network of bulk water and at the same time decrease the solubility of biomolecules. In contrast chaotropic ions (e.g. nitrate ion) supposedly break the hydrogen-bonding network of bulk water and increase the solubility of biomolecules. Recently, the interfacial tension at the protein-water interface was shown to play a central role in the Hofmeister phenomena.[5] Not only salts, but other chemical agents can also denature proteins. Bennion and Daggett simulated the ureainduced denaturation of chymotrypsin and suggested that the solvent plays various roles in the process. Most importantly, the structure and dynamics of the solvent changed in the solution, and intrusion of the solvent molecules into the hydrophobic core of chymotrypsin was responsible for diminishing the hydrophobic effect and encouraging solvation of the core and thereby changing the intramolecular hydrogen bond network in the protein.[6]

Generally, it is accepted that different levels of hydration occur at a biomolecule. In the first hydration layer water interacts with the external surface of the protein through directional hydrogenbonding (H-bonding) interaction especially on the hydrophilic surface of the protein, while on the hydrophobic surface of the protein the topology, roughness and spatial constrains of the surface orient the water molecules. As a consequence, the hydrogen-bonded properties of water is influenced significantly by the surface properties of macromolecules resulting in increased mean residence time[7-12] and 10-20\% increase of the density of water molecules[13,14] compared to the bulk phase. However, a molecular dynamics study on myoglobin, also showed that only those water molecules have very long residence times that are found in cavities and clefts of the protein; other hydration sites of the protein are characterized by residence times similar to the bulk phase.[15] The water-water hydrogen bonding in the first layer forms a spanning, peptide homogeneously enveloped, percolated network, while lack of biological functions is always connected to the broken 
(not percolated) H-bonded network in the first layer.[16] Recent terahertz spectroscopic

60 measurements, a method sensitive to the collective motion of water molecules, indicate that protein disturb the water structure beyond the 1-2 water layers as previously thought.[17] The radius of the dynamic hydration shell was greater than $10 \AA$ for the studied proteins and correlated well with the dipole moment of the protein.[18]

It is obvious from the above overview that gaining a better understanding of the hydration layer structure around proteins could contribute to our understanding of various processes involving protein-solvent interactions such as protein folding and unfolding. A possible way to study the structure of water is graph theory, which has recently been applied to the hydrogen bond network in various solutions and mixtures, e.g. of water, methanol and ethanol solutions adsorbed in microporous silicalite-1[19], of ion aggregates in different high salt solutions[20], and of highly concentrated renal osmolyte solutions.[21] Recently, we studied the mixtures of water and formamide, the simplest model of the peptide bond, and showed that these two compounds form microhomogeneous mixtures, in which the number of hydrogen bonds formed by water and formamide are very similar.[22]

When graph theory is used to study the structure (i.e. hydrogen bond network) of water, the network of interactions is mapped into a graph.[23] The vertices of the graph correspond to the water molecules and the edges to the hydrogen bonds formed by the water molecules. Once the hydrogen bond network is mapped into a graph, a thorough statistical analysis can be carried out in order to get insight into the water structure.

In the present work we extend this theory to explore the hydrogen bond network around a protein to obtain a better understanding of its structure and how it changes from the surface of the protein towards the bulk phase, and investigate how molecular dynamics simulations can give more insight into recent findings by terahertz spectroscopic measurements that proteins disturb the water structure beyond 1-2 water layers. Furthermore, we explore the effect of salt concentration on the properties of the hydrogen bond network around a protein. We chose insulin as a model protein because of its (1) small size (2) importance in human health (3) and as it has a balanced distribution of hydrophobic and hydrophilic patches on its surface. $\mathrm{NaCl}$ was selected as a co-solute to the protein, because $\mathrm{Na}^{+}$ has a relatively high concentration in the cytosole compared to divalent cations and it has a weak hydrate sphere ordering capacity and $\mathrm{Cl}^{-}$is by far the most common anion in living organisms.

First we carried out a series of molecular dynamics simulations at different salt concentrations on 
91 the simulations, we determined the network of hydrogen bonds and transformed it to a graph. Finally,

92 a thorough statistical analysis of the properties of the obtained graphs (hydrogen bond neighbor

93 number and the interaction energy between hydrogen-bonded water molecules) were carried out.

94 Importantly, we present here a simpler approach to define solvent layers around the protein compared

95 to those that have been described in the literature,[24-26] and investigate their hydrogen bond

96 properties layer by layer, which enables us to compare the structure and hydrogen bond properties

97 of these layers to those of reference solutions, which do not include the protein molecule. This

98 methodology enables us to characterize the effect of the protein molecule on the hydrogen bond

99 network and to study the structure of its hydration sphere in a statistical way. 
101 Molecular dynamics simulations (MD). The crystal structure of the monomer, which is the active 102 form of human insulin (PDB code 3I40[27]) was used as a starting structure for the MD simulations.

103 Disulfide bonds were created between Cys6,chainA-Cys 11,chainA, Cys7,chain A-Cys7,chainB and Cys20,chainA-

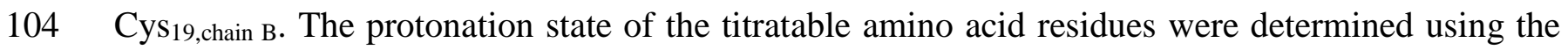
$105 \mathrm{H}++$ webserver version 3.2.[28-30] Based on the estimated $\mathrm{pK}_{\mathrm{a}}$ values the His 5 residue was doubly 106 protonated in chain B and after visual analysis of their surroundings all other histidine residues were 107 protonated on the $\varepsilon$ nitrogen atom. The CHARMM-GUI webserver was used for the system setups 108 and generation of the input files.[31], the NAMD software package[32] with the CHARMM27 force 109 field[33] for the minimization of the structure and dynamics simulations. Hydrogen atoms were 110 added using the standard CHARMM protocol.[34] We chose the TIP3P water model, which is a 111 simple 3-point rigid water model, to simulate water as the non-bonded parameters of protein atom 112 types in the CHARMM27 force field were determined to be in line with the TIP3P water model. As 113 a consequence, when the CHARMM program package is used for modelling proteins the TIP3P 114 water model is by far the most frequently used water model, yielding a reliable description of 115 proteins. Therefore, the protein was solvated by TIP3P water molecules arranged in an octahedral 116 shape with $15 \AA$ edge distances. Three differently solvated protein systems were prepared. One 117 contained only one sodium ion in order to generate a neutral system, while two other systems 118 contained sodium and chloride ions in 0.5 and 1.5 molar concentrations. Although the cytosolic 119 concentration of these ions is much lower, we have chosen these relatively high concentrations to 120 obtain improved statistics for the effect of the desalting in the simulation. The ions were placed by a 121 Monte Carlo approach. Reference systems of the salt solutions with $0 \mathrm{M}, 0.5 \mathrm{M}$ and $1.5 \mathrm{M}$ 122 concentrations were also prepared; these did not include the protein molecule. Afterwards, each 123 system was minimized for 10000 steps to eliminate bad initial contacts, followed by a 50 ps long 124 NVT equilibration simulations at $303.15 \mathrm{~K}$ temperature. Then $5 \mathrm{~ns}$ long NPT Langevin MD 125 simulations were carried out applying 2 fs step size with collecting configurations from every ps. All 126 bonds in the molecules involving hydrogen atoms were kept fixed with the SHAKE[35] algorithm.

127 Periodic boundary conditions (PBC) were used to handle boundary effects. The temperature was set 128 to $303.15{ }^{\circ} \mathrm{C}$ in all simulations. The equilibration of the systems was reached by means of 129 temperature reassignment. All of the velocities of the atoms in the systems were periodically 130 reassigned in order to set the desired temperature. Therefore, in every 500 steps the temperature was 131 rescaled during equilibration. The Constant Temperature Control making use of Langevin dynamics. 132 was applied together with the Nose-Hoover Langevin piston pressure control with the target pressure 
set to 1.01325 bar. As the random initial velocity distribution used in the MD simulation could

134 influence the obtained results, all MD simulations were carried out with three different initial velocity distributions yielding 3-3 parallel trajectories for all systems studied.

\section{Analysis}

137 Hydrogen bonds. Two water molecules were regarded hydrogen bonded if the $\mathrm{H} \cdots \mathrm{O}$ distance 138 between the two molecules was smaller than $2.5 \AA$ and $\mathrm{O}-\mathrm{H} \cdots \mathrm{O}$ angle was larger than $120^{\circ}$. This criteria are reasonable for protein simulations where correlation between the distance and the angle criteria have already been shown.[36] However, we have checked the dependency of our results on this definition by analyzing the trajectories obtained for the protein solvated in $0.5 \mathrm{M} \mathrm{NaCl}$ solution by setting the $\mathrm{O}-\mathrm{H} \cdots \mathrm{O}$ angle criterion to $130^{\circ}$ and $145^{\circ}$ as well.

143 The average hydrogen bond number, $\mathrm{N}_{\mathrm{HB}}$, was calculated by averaging the number of hydrogen bonds over the trajectory and over all molecules (Eq. 1):

$$
\mathrm{N}_{\mathrm{HB}}=\frac{<\sum_{\mathrm{i}=1}^{\mathrm{N}} \mathrm{N}_{\mathrm{HB}, \mathrm{i}}>}{2 \mathrm{~N}}
$$

145 where $\mathrm{N}_{\mathrm{HB}, \mathrm{i}}$ is the number of hydrogen bonds around water molecule $\mathrm{i}, \mathrm{N}$ is the number of water molecules in the simulated box and $<>$ denotes averaging over all snapshots.

147 We calculated the hydrogen bond energy between water molecules $\mathrm{i}$ and $\mathrm{j}\left(\mathrm{E}_{\mathrm{HB}, \mathrm{ij}}\right)$ for each hydrogen 148 bond using the TIP3P force field following the common practice[37] to identify the hydrogen-bond 149 energy with the interaction energy of the H-bonded molecular pair, even if the H-bond energy cannot 150 be separated from the rest of the pair interaction energy. It was averaged over all hydrogen bonded 151 pairs and over the whole length of the trajectory to yield the average hydrogen bond energy $\left(\mathrm{E}_{\mathrm{HB}}\right)$ 152 using the following equation:

$$
\mathrm{E}_{\mathrm{HB}}=\frac{<\sum_{\mathrm{i}=1}^{\mathrm{N}} \sum_{\mathrm{j}=1}^{\mathrm{N}_{\mathrm{HB}, \mathrm{i}}} \mathrm{E}_{\mathrm{HB}, \mathrm{ij}}>}{<\sum_{\mathrm{i}=1}^{\mathrm{N}} \mathrm{N}_{\mathrm{HB}, \mathrm{i}}>}
$$

$153 \mathrm{E}_{\mathrm{HB}, \mathrm{ij}}$ is the energy of the hydrogen bond between molecules $\mathrm{i}$ and $\mathrm{j}$ and the rest of the notation 154 corresponds to Eq. 1.

156 Definition of solvation layers. As experimental data indicate that proteins disturb considerably the 157 water structure around them, we have developed a methodology to define hydration layers around 158 the protein (Fig. 1). Molecules on the surface of the protein (first layer) were defined according to 159 two rules: (1) water molecules were assigned to interact with the hydrophilic surface of the protein 
160 if any of the following distances $\left(\mathrm{O}_{\text {Water }}-\mathrm{H}_{\text {Protein }}\right.$ or $\mathrm{H}_{\text {Water }}-\mathrm{O}_{\text {Protein }}$ or $\left.\mathrm{H}_{\text {Water }}-\mathrm{N} / \mathrm{S}_{\text {Protein }}\right)$ was smaller than

$1612.5 \AA$ and (2) water molecules in the first layer interacting with the hydrophobic surface of the protein

162

163

164

165

166

167

168

169

170

171

172

173

174

175

176

177

178

179

180 were defined as having a $\mathrm{C}_{\text {Protein- }} \mathrm{O}_{\text {Water }}$ (abbreviated to $\mathrm{C}-\mathrm{O}$ distance from now on) distance smaller than $4.5 \AA$ and not satisfying rule (1). Application of these two rules provided us with a continuous first hydration layer over the surface of the protein. The second hydration layer consisted of the water

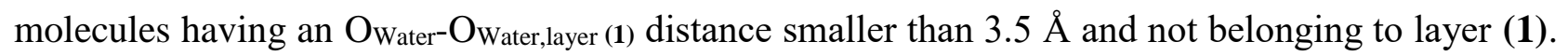

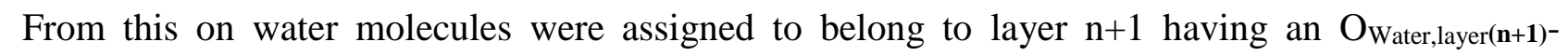

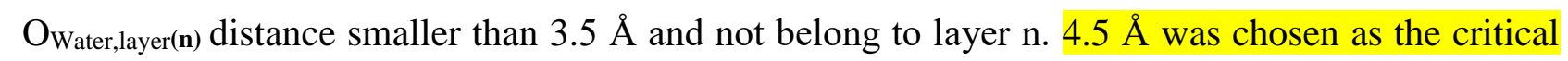
C-O distance, because it is very close to the minimum of the partial radial distribution function of the $\mathrm{C}-\mathrm{O}$ distance in liquid methanol or of the $\mathrm{C}-\mathrm{O}_{\text {water }}$ distance in water-methanol mixtures. It is also the typical $\mathrm{C}-\mathrm{O}$ distance between the carbon atom of $\mathrm{CH}_{3}$ groups and the oxgen atom of the water molecule closest to them. However, we have tested the dependency of our results on this distance, and the analyses were performed using 4.0, 4.25, 4.5 and $5 \AA$ criteria as well in the case of insulin system solvated in $0.5 \mathrm{M} \mathrm{NaCl}$ solution.

\section{Results}

Protein behaviour at different salt concentrations. We compared the behavior of the proteins at various salt concentrations in the MD simulations. The results have been averaged over all snapshots of the productive part of the MD simulation and over the three parallel trajectories carried out at identical salt concentrations. In order to check the stability of the protein conformation during the simulations we have calculated the Root Mean Square Deviation (RMSD) of the atomic positions.

$$
R M S D=\sqrt{\frac{\sum_{i=1}^{N}\left(r_{i}\left(t_{1}\right)-r_{i}\left(t_{2}\right)\right)^{2}}{N}}
$$

181 where $\mathrm{N}$ is the number of atoms and $\mathrm{r}_{\mathrm{i}}(\mathrm{t})$ is the position of atom $\mathrm{i}$ at time $\mathrm{t}$. In all cases $\mathrm{t}_{1}$ was the

182 position of the protein heavy atoms after the heating up of the system. The comparison of the protein

183 RMSD values for all trajectories is shown in Table 1, decomposed according to the various structural

184 elements found in insulin. The structural elements are shown in Fig. 2. While the obtained RMSD

185 values are similar in the cases of the neutralized and $0.5 \mathrm{M} \mathrm{NaCl}$ systems, slightly lower RMSD

186 values have been observed in the case of the $1.5 \mathrm{M} \mathrm{NaCl}$ system. This could originate from the fact

187 that in this reasonably concentrated $1.5 \mathrm{M}$ solution ions have a stronger tendency to absorb on the

188 surface of the protein and slightly stabilize its structure as the ion-surface interaction is much stronger

189 than the H-bonds between the surface and the water molecules. 
The RMSD values show that insulin remained in its natural conformation along the MD trajectory.

191 We were also interested to check how other parameters, the radius of gyration and the geometric 192 moment tensor changed along the trajectory, as they could also give insight into structural changes

193 of the protein. Nayar et all showed that the gyration radius of a globular protein (of the 16-residue $194 \beta$-hairpin fragment of the 2GB1 protein) shows a remarkable (at least 1.5-2 $\AA$ ) difference between 195 its folded and unfolded states.[38] The gyration radius of insulin (10.1 $\AA \pm 0.1 \AA)$ was quite stable 196 during the whole simulation in all three solution. indicating the stability of the protein structure.

197 Globular proteins in their native conformation, like insulin, have close-packed structures with quite 198 high number densities and a well-defined shape in the solution. Characterization of the shape of 199 proteins has been in the focus of intense scientific interest for many years. One of the applicable 200 method for the description of the shape of a whole protein uses simple ellipsoids. Here, we apply for 201 this purpose the size of the three main axes of the geometry moment tensor, which is calculated with 202 a similar mathematical construction, as the moment of the inertia tensor, with the difference that each 203 point (i.e. the coordinates of all heavy atoms) is assigned the same mass: a mass of unity, instead of 204 the real mass of the atom. The values of the size of the three main geometrical moments as a function 205 of time are presented in Fig. 3 in the case of the 1.5 M solution, but we obtained very similar graphs 206 in the case of the other two concentrations as well. The figure shows that the overall shape of 207 the protein does not change significantly during the simulation time. This method also enables us to 208 characterize the shape of the solvation layers. We performed this for this first three hydration layers 209 together, which is also shown in Fig. 3. It is obvious that the shape of the solvation layers closely 210 follow that of the protein, with longer axes.

211 Number of the water molecules in the solvation layers. We determined the number of water 212 molecules in each shell around the protein (see Table 2). As expected as a function of layer number 213 the number of water molecules increases significantly at each salt concentration as we go farther 214 away from the protein. It seems that there is no significant difference between the number of water 215 molecules in the first and second shell around the protein in the case of the neutralized system and 216 the $0.5 \mathrm{M} \mathrm{NaCl}$ concentration system, while there seem to be less water molecules around 217 hydrophilic surface of the protein in the simulations with the highest salt concentration. Here it is 218 worth noting, that $\mathrm{Na}^{+}$and $\mathrm{Cl}^{-}$are neither chaotropic nor cosmotropic, thus they have no strong 219 tendency either to be attracted nor to be repelled from the surface of proteins. We examined the 220 number of water molecules associated to the hydrophilic and hydrophobic surface of the protein and 221 it is seen that at all concentrations the number of water molecules at the hydrophobic surface remains 222 identical, but decreases slightly at the hydrophilic surface. There are most likely two different reasons 
223 for this: (1) the solvent accessible surface area of the protein slightly decreases and as such fewer 224 water molecules can access it (2) with increasing salt concentration, the "place" of several water 225 molecules is taken over by ions, which can also occur especially on the hydrophilic surface of the 226 protein. This finding is in accordance with the expectation that ions are repelled from 227 hydrophobic/non-polar regions. As we go farther away from the protein surface we find an increasing 228 difference among the number of molecules in a given shell with increasing salt concentration due to 229 the presence of an increasing number of ions.

230 Radial distribution function. The oxygen-oxygen $\left(\mathrm{O}_{\text {water }}-\mathrm{O}_{\text {water }}\right)$ partial radial distribution function 231 (RDF) can be used for characterization of the structure of water around the protein surface.[39,40] 232 We calculated two different types of radial distribution functions to evaluate the extent of the water 233 structure around protein surface. In one of them we calculated the RDF for the interactions between 234 the water molecules in the first layer- and all other water molecules in layers 2-4 to obtain the surface 235 distribution function (SDF). In the other case water-water RDF was calculated only for water 236 molecules in the first layer. Unfortunately, from our simulations we do not know a priori the exact 237 density of water in the simulation box and we could not properly calculate the excluded volume 238 effect, so the raw $\mathrm{O}_{\text {water }}-\mathrm{O}_{\text {water }}$ density distribution was determined using the spherical average 239 method according to Eq. 4.:

$$
\rho(r)=\frac{N(r)}{\left(4 \pi r^{2} d r\right)}
$$

240 where $\mathrm{N}$ denotes the number of particles. It can be seen from both Figs. 4 and $\mathbf{5}$ that the curvature of 241 the raw density distribution functions is the same for all interaction types, thus we can assume that 242 the necessary corrections to account for the excluded volume effect would be the same in all cases, 243 thus we can obtain correct trends from the raw distribution functions as well.

244 In Fig. 4 the surface distribution functions are shown for the interactions between hydrophobic- and 245 hydrophilic-all other water molecules in layers 2-4 cases. We cannot detect any large changes on 246 these SDFs as a function of salt concentration. The first peak on these SDF is around $2.8 \AA$ and it is 247 significantly more pronounced for the hydrophobic surface water-all water case.

248 The raw density distribution functions corresponding to the first layer-first layer structure are 249 presented in Fig. 5. On the insect we presented the long range behavior of these functions, which 250 show that the long range behavior of these function is the same for different interaction types: 251 hydrophilic-hydrophilic (Hy-Hy), hydrophobic-hydrophobic (Hyb-Hyb) and hydrophilic252 hydrophobic (Hy-Hyb); thus these functions can be compared. The H-bonded structure, which can 
be characterized by the first peak at around $2.8 \AA$ is more pronounced in the Hyb-Hyb case, which is in good agreement with results of statistical analysis of H-bond strength (see below). The shape of the first peak of the hydrophilic-hydrophilic (Hy-Hy) RDF is considerably deviates from the other two (Hyb-Hyb,Hy-Hyb) RDFs, especially in the range of 3.2-4.5 $\AA$. This difference in shape indicates (1) the uniformity of the interaction between water molecules over the hydrophobic surface of the water molecules (2) larger differences in interaction strengths between water molecules over the hydrophilic surface of the water. This implies that water molecules behave differently over the hydrophobic and hydrophilic surface of the protein and supports the hypothesis of structural and dynamical heterogeneity of the surface.

Average hydrogen bond number. Next, we determined the average number of hydrogen bonds formed by water molecules in the reference aqueous solutions not including the protein, then we analyzed the hydrogen bonds formed by water molecules in the protein-containing systems. In the latter case we determined how many hydrogen bonds are formed between water molecules in the same layer, and between two layers as well. In the case of the first hydration layer the data was even further decomposed that we could see the effect of the protein on the hydrogen bond network. The data is collected in Table 3. In the reference solutions, the average hydrogen bond number in the pure water and at $0.5 \mathrm{M} \mathrm{NaCl}$ solution is 3.4 which agrees very well with the results of earlier studies.[23,41] At the highest salt solution, the average hydrogen bond number is decreased to 3.14, which clearly originates from the fact that at such a high salt concentration, significant number of hydrogen bonding sites of the water molecules are occupied by the solute ions, i.e. the water molecule itself belongs with an increasing probability to the solvent sphere of an ion.

Water molecules in the $1^{\text {st }}$ solvation shell establish significantly lower number of hydrogen bonds with other water molecules than in the bulk phase (e.g. $\mathrm{n}_{\mathrm{HB}, \mathrm{ref}}=3.40$ at $\left.0.5 \mathrm{M}\right)$, but this effect is overcompensated by the hydrogen bonds established with the protein. In the $2^{\text {nd }}$ shell significantly smaller number of hydrogen bonds are found compared to the bulk phase or the other solvation layers. From the $3^{\text {rd }}$ shell the average hydrogen bond number and the structure of the hydrogen bond network begins to resemble to the reference systems, although the total number of hydrogen bonds is slightly larger. As a result of the increase of the salt concentration the number of the hydrogen bonds $\left(\mathrm{N}_{\mathrm{HB}}\right)$ decreases, and water molecules in the $3^{\text {rd }}$ and $4^{\text {th }}$ solvation shells form similar number of hydrogen bonds than found in the reference systems (aqueous solutions with identical salt concentration). (Here we would like to note that in the reference systems the hydrogen bonds among water molecules that are not in the coordination sphere of sodium or chloride ions are stronger by 1.7- $2.5 \mathrm{~kJ} / \mathrm{mol}$ than the average hydrogen bond number averaged over all water molecules.) Our 
data show that the number of hydrogen bonds in the $1^{\text {st }}$ solvation shell are considerably larger than in the $2^{\text {nd }}$ layer. The number of hydrogen bonds $\left(\mathrm{N}_{\mathrm{HB}}\right)$ formed only within the $1^{\text {st }}$ shell is around 1.8 and the occurrence of a few very large clusters in the cluster size distribution indicate the formation of a percolated network (i.e. a continuous network of interconnected clusters) in good agreement with previous data of Brovchenko et al.[42] We performed the same cluster analysis for solvation layers 2, 3 and 4 separately, taking into account only the in-layer H-bonds, but in these layers the water molecules do not form a percolated network in any of these layers. The average number of hydrogen bonds formed among water molecules in the $2^{\text {nd }}$ solvation shell is remarkably small. This is due to the fact that while water molecules in the $1^{\text {st }}$ layer orient themselves to form as many hydrogen bonds with the protein as possible, the $3^{\text {rd }}$ layer orients itself towards bulk water, but water molecules in the $2^{\text {nd }}$ layer cannot easily accommodate themselves to establish an optimal number of hydrogen bonds. This is best regarded as a transient layer whose properties are determined by a combined effect of the adaptation to the shape of the protein molecule and to the network of water molecules in the bulk solvent.

We have checked the dependence of the results on the applied H-bond criteria. In Table 4 we have collected the data obtained with various $\mathrm{C}-\mathrm{O}$ distances used to allocate water molecules over the hydrophobic surface of the protein. It can be observed that the $\mathrm{C}-\mathrm{O}$ distance primarily influences the average $\mathrm{H}$-bond neighbor number in the first shell, taking longer distance the $\mathrm{n}_{\mathrm{HB}}$ slightly increases. The same effect is observed in the second shell although to a lesser extent, but no effect can be seen in the case of the farther layers. The reason observed for the first two layers arises from the fact that by changing the $\mathrm{C}-\mathrm{O}$ distance the number of water molecules slightly changes over the hydrophobic surface of the protein, some molecules may be assigned to layer 1 or layer 2 depending on the exact $\mathrm{C}-\mathrm{O}$ distance, but at larger distances this effect diminishes. However, it is important to emphasizes that our conclusions do not depend on the exact value of the C-O distance between 4-5 $\AA$.

310 We have also investigated how the obtained results depend on the angle criteria used to determine 311 hydrogen bonds. The data in Table 5 show that with using a larger angle criterion, the average $\mathrm{H}-$ bond neighbor number slightly decreases as only more "perfect" H-bonds are identified as H-bonds (i.e. a smaller number of them is found), but the observed trends do not change at all.

\section{Changes in hydrogen bond energy as a function of the distance from the surface of the protein.}

315 For the characterization of the hydrogen bonded interaction we can use the strength of the hydrogen 316 bond. This strength is identified by the interaction energy of the two hydrogen bonded water 317 molecules. In order to sign the perturbation effect of the protein we have investigated the average 318 hydrogen bond energies in each layer and between the layers. Student's t-test, a rigorous statistical 
probe, was used to prove that the observed differences between the obtained values are significant.

320 Data in Table 6 show that the strongest hydrogen bonds between water molecules are formed in the pure liquid water reference system, and addition of solutes (either protein or salt) decreases the hydrogen bond strength, and this decrease becomes more significant with increasing salt concentration. Furthermore, Table 7 shows that changing the angle criteria does not influence the observe trends. We can decompose this value to calculate the hydrogen bond energy strength between water molecules associated to the hydrophilic or to the hydrophobic surface of the protein. It is clear from these data that hydrogen bonds among water molecules at the hydrophobic surface are much stronger than at the hydrophilic surface. This is due to the protein-water interactions at the hydrophilic surface, where water molecules orient themselves to interact mainly with protein surface groups. However, over the hydrophobic surface water molecules mainly interact with each other leading to more optimal hydrogen bonded arrangements. Furthermore, the average hydrogen bond strength in the first layer is mainly governed by interactions of the hydrophobic water molecules, and suggests that the interaction between a hydrophobic and hydrophilic water molecule is also very strong. These results are in a good agreement with the differences of the water-water partial radial distribution functions calculated for the various parts (hydrophobic or hydrophilic) of the protein surface (Fig. 5).

We also observe considerable difference in the strength of the interactions between and in the layers (e.g. in the case of the $0.5 \mathrm{M}$ system: inside the $2^{\text {nd }}$ layer $-14.88 \mathrm{~kJ} / \mathrm{mol}$ and between the $2^{\text {nd }}$ and $3^{\text {rd }}$ layers $-15.34 \mathrm{~kJ} / \mathrm{mol}$, respectively), which indicates that the perturbation effect caused by the protein shape is more pronounced in interactions in the layers than between the layers. This phenomenon is in the $4^{\text {th }}$ and $5^{\text {th }}$ layers begin to disappear. In these layers the strength of the hydrogen bonds is getting closer and closer to the values observed in the reference systems. (Here we would like to note that in the reference systems the hydrogen bond strength between water molecules that are not in the coordination sphere of sodium or chloride ions is only $0.08-0.13 \mathrm{~kJ} / \mathrm{mol}$ stronger than the average hydrogen bond strength averaged over all water molecules.) Due to the salt concentration, the average hydrogen bond strength decreases in the same way as in the reference systems.

\section{Relevance of our results to physiological solutions}

347 After having examined numerous properties of the hydrogen bond network around insulin, used as 348 a model protein, it is worth putting our results into a wider context and consider their relevance to 349 physiological and other solutions. The data show that proteins disturb the H-bond network at least 350 up to five water layers, which imply a large volume of water and reasonably large distances between 351 the protein molecules. We calculated the concentration of the protein in our systems to be $8.360^{*} 10^{-}$ 
353 As an example of a physiologically relevant solution we could consider blood, whose typical protein concentration can be estimated the following way. The most abundant proteins in blood are albumins (constituting about $55 \%$ of blood proteins), which are present in 3-5 g/dL concentration. The molar weight of albumins is around $65 \mathrm{kDalton}$ (or $65000 \mathrm{~g} / \mathrm{mol}$ ). Taking $5 \mathrm{~g} / \mathrm{dL}$ concentration this would yield a concentration of $7.69 * 10^{-4} \mathrm{M}$. Most other proteins present in blood have a much higher molecular weight (e.g. the molar mass of globulins is between 93-1193 kDalton), thus they increase only slightly the molar protein concentration of blood or they are present in much smaller quantities e.g. typical blood level of insulin between meals is $57 * 10^{-4}$ M.[43] This implies that blood is a slightly more diluted protein solution than the insulin solution studied by us, thus the 5 water layers is likely to be present around protein in blood and most likely in other physiologically relevant solutions. It is worth keeping in mind, though, that blood and other physiological solutions contain a variety of other co-solutes, e.g. sugars and other small molecules, which also influence the water network in them. Furthermore, from smaller peptides one may prepare much more concentrated solutions, where the individual peptide molecules may be closer to each other and there could be less than 8-10 hydration layers between two peptide molecules (i.e. 5 layers belonging to peptide 1 and five layers belonging to peptide would yield a separation of 10 layers). In this case it is very likely that proteins would seriously influence each other's solvation spheres, and in this case our conclusions would not be valid. A separate study would be needed to study the effect of peptide concentration on the structure of the hydration shells of peptides.

\section{Conclusions}

373 In this work we have studied the topological properties of the water layers around protein molecules 374 as a function of sodium chloride concentration, using insulin as a model system. Our statistical 375 analysis shows a significant difference among the hydrogen-bonded properties of water molecules 376 in the first, second and farther solvation layers. We can also show that water molecules over the 377 hydrophilic and hydrophobic surface of the protein possess slightly different H-bonding properties, 378 supporting the hypothesis of structural and dynamical heterogeneity of the water molecules over the 379 protein surface. The effect of the protein on the hydrogen bonded water network exist at least up to 3804 layers, which is in accordance with recently reported sub-terahertz spectroscopic measurements.

\section{Acknowledgements}

382 A.L. thanks the financial support for the Richter Gedeon Talentum Foundation. J.O. was supported 383 by the Bolyai János Research Scholarship and by NKFIH Grant No. 115503. I.B. was supported by 384 OTKA Grant No. 108721. 


\section{References}

1 Frauenfelder H, Sligar SG \& Wolynes PG (1991) The energy landscapes and motions of proteins. Science 254, 1598-1603.

2 Austin RH, Beeson KW, Eisenstein L, Frauenfelder H \& Gunsalus IC (1975) Dynamics of ligand binding to myoglobin. Biochemistry 14, 5355-5373.

3 Hofmeister F (1888) On the understanding of the effect of salts. Second report. On regularities in the precipitating effect of salts and their relationship to their physiological behavior. NaunynSchmiedebergs Arch. Exp. Pathol. Pharmakol 24, 247-260.

4 Baldwin RL (1996) How Hofmeister ion interactions affect protein stability. Biophys. J. 71, 2056-2063.

5 Bogár F, Bartha F, Násztor Z, Fábián L, Leitgeb B \& Dér A (2014) On the Hofmeister Effect: Fluctuations at the Protein-Water Interface and the Surface Tension. J. Phys. Chem. B 118, 8496-8504.

6 Bennion BJ \& Daggett V (2003) The molecular basis for the chemical denaturation of proteins by urea. Proc. Natl. Acad. Sci. U. S. A. 100, 5142-5147.

7 Otting G, Liepinsh E \& Wüthrich K (1991) Protein hydration in aqueous solution. Science 254, 974-980.

8 Denisov VP \& Halle B (1996) Protein hydration dynamics in aqueous solution. Faraday Discuss. 103, 227-244.

9 Wiesner S, Kurian E, Prendergast FG \& Halle B (1999) Water molecules in the binding cavity of intestinal fatty acid binding protein: dynamic characterization by Water $17 \mathrm{O}$ and $2 \mathrm{H}$ magnetic relaxation dispersion. J. Mol. Biol. 286, 233-246.

10 Henchman RH \& McCammon JA (2002) Structural and dynamic properties of water around acetylcholinesterase. Protein Sci. 11, 2080-2090.

11 Pal SK \& Zewail AH (2004) Dynamics of water in biological recognition. Chem. Rev. 104, 2099-2123.

12 Russo D, Hura G \& Head-Gordon T (2004) Hydration dynamics near a model protein surface. Biophys. J. 86, 1852-1862.

13 Rossky PJ \& Cheng Y-K (1998) Surface topography dependence of biomolecular hydrophobic hydration. Nature 392, 696-699.

14 Makarov V, Pettitt BM \& Feig M (2002) Solvation and hydration of proteins and nucleic acids: A theoretical view of simulation and experiment. Acc. Chem. Res. 35, 376-384.

15 Makarov VA, Andrews BK, Smith PE \& Pettitt BM (2000) Residence Times of Water Molecules in the Hydration Sites of Myoglobin. Biophys. J. 79, 2966-2974.

16 Oleinikova A \& Brovchenko I (2011) What Determines the Thermal Stability of the HydrogenBonded Water Network Enveloping Peptides? J. Phys. Chem. Lett. 2, 765-769.

17 Ebbinghaus S, Kim SJ, Heyden M, Yu X, Heugen U, Gruebele M, Leitner DM \& Havenith M (2007) An extended dynamical hydration shell around proteins. Proc. Natl. Acad. Sci. 104, 20749-20752. 
18 Sushko O, Dubrovka R \& Donnan RS (2015) Sub-terahertz spectroscopy reveals that proteins influence the properties of water at greater distances than previously detected. J. Chem. Phys. 142, 55101.

19 Wang C-H, Bai P, Siepmann JI \& Clark AE (2014) Deconstructing Hydrogen-Bond Networks in Confined Nanoporous Materials: Implications for Alcohol-Water Separation. 118, 1972319732.

20 Choi J-H \& Cho M (2014) Ion aggregation in high salt solutions. II. Spectral graph analysis of water hydrogen-bonding network and ion aggregate structures. J. Chem. Phys. 141, 154502.

21 Lee H, Choi J-H, Verma PK \& Cho M (2015) Spectral Graph Analyses of Water HydrogenBonding Network and Osmolyte Aggregate Structures in Osmolyte-Water Solutions. J. Phys. Chem. B 119, 14402-14412.

22 Bakó I, Oláh J, Lábas A, Bálint S, Pusztai L \& Bellissent Funel MC (2017) Water-formamide mixtures: Topology of the hydrogen-bonded network. J. Mol. Liq. 228, 25-31.

23 Bakó I, Bencsura Á, Hermannson K, Bálint S, Grósz T, Chihaia V \& Oláh J (2013) Hydrogen bond network topology in liquid water and methanol: a graph theory approach. Phys. Chem. Chem. Phys. 15, 15163-15171.

24 Mezei M (2003) A new method for mapping macromolecular topography. J. Mol. Graph. Model. 21, 463-472.

25 Willard AP \& Chandler D (2010) Instantaneous Liquid Interfaces. J. Phys. Chem. B 114, 1954 1958.

26 Sega M, Kantorovich SS, Jedlovszky P \& Jorge M (2013) The generalized identification of truly interfacial molecules (ITIM) algorithm for nonplanar interfaces. J. Chem. Phys. 138, 44110.

27 Timofeev V., Chuprov-Netochin R., Samigina V., Bezuglov V., Miroshnikov K. \& Kuranova IP (2010) X-ray investigation of gene-engineered human insulin crystallized from a solution containing polysialic acid. Acta Crystallogr.,Sect.F 66, 259-263.

28 Anandakrishnan R, Aguilar B \& Onufriev A V (2012) H++ 3.0: automating pK prediction and the preparation of biomolecular structures for atomistic molecular modeling and simulations. Nucleic Acids Res. 40, W537-541.

29 Myers J, Grothaus G, Narayanan S \& Onufriev A (2006) A simple clustering algorithm can be accurate enough for use in calculations of pKs in macromolecules. Proteins 63, 928-938.

30 Gordon JC, Myers JB, Folta T, Shoja V, Heath LS \& Onufriev A (2005) H++: a server for estimating pKas and adding missing hydrogens to macromolecules. Nucleic Acids Res. 33, W368-371.

31 Jo S, Kim T, Iyer VG \& Im W (2008) CHARMM-GUI: a web-based graphical user interface for CHARMM. J. Comput. Chem. 29, 1859-1865.

32 Phillips JC, Braun R, Wang W, Gumbart J, Tajkhorshid E, Villa E, Chipot C, Skeel RD, Kalé L \& Schulten K (2005) Scalable molecular dynamics with NAMD. J. Comput. Chem. 26, 17811802.

33 MacKerell AD, Banavali N \& Foloppe N (2000) Development and current status of the CHARMM force field for nucleic acids. Biopolymers 56, 257-265.

34 Brooks BR, Brooks CL, Mackerell AD, Nilsson L, Petrella RJ, Roux B, Won Y, Archontis G, 
Bartels C, Boresch S, Caflisch A, Caves L, Cui Q, Dinner AR, Feig M, Fischer S, Gao J, Hodoscek M, Im W, Kuczera K, Lazaridis T, Ma J, Ovchinnikov V, Paci E, Pastor RW, Post CB, Pu JZ, Schaefer M, Tidor B, Venable RM, Woodcock HL, Wu X, Yang W, York DM \& Karplus M (2009) CHARMM: the biomolecular simulation program. J. Comput. Chem. 30, 1545-1614.

35 Ryckaert J-P, Ciccotti G \& Berendsen HJ. (1977) Numerical integration of the cartesian equations of motion of a system with constraints: molecular dynamics of n-alkanes. $J$. Comput. Phys. 23, 327-341.

36 De Loof H, Nilsson L \& Rigler R (1992) Molecular dynamics simulation of galanin in aqueous and nonaqueous solution. J. Am. Chem. Soc. 114, 4028-4035.

37 Wendler K, Thar J, Zahn S \& Kirchner B (2010) Estimating the Hydrogen Bond Energy. J. Phys. Chem. A 114, 9529-9536.

38 Nayar D \& Chakravarty C (2014) Sensitivity of local hydration behaviour and conformational preferences of peptides to choice of water model. Phys. Chem. Chem. Phys. 16, 10199-10213.

39 Rani P \& Biswas P (2014) Shape dependence of the radial distribution function of hydration water around proteins. J. Phys. Condens. Matter 26, 335102.

40 Rani P \& Biswas P (2015) Local Structure and Dynamics of Hydration Water in Intrinsically Disordered Proteins. J. Phys. Chem. B 119, 10858-10867.

41 Abhishek Rastogi AKG and SJS (2011) Hydrogen bond interactions between water molecules in bulk liquid, near electrode surfaces and around ions InTech.

42 Oleinikova A, Brovchenko I, Smolin N, Krukau A, Geiger A \& Winter R (2005) Percolation Transition of Hydration Water: From Planar Hydrophilic Surfaces to Proteins. Phys. Rev. Lett. 95, 247802.

43 Iwase H, Kobayashi M, Nakajima M, Takatori T, Rindfrey H, Lang H, Leybold K, Rick W \& Staudinger HJ (2001) The ratio of insulin to C-peptide can be used to make a forensic diagnosis of exogenous insulin overdosage. Forensic Sci. Int. 115, 123-127. 
495 Table 1. Average protein RMSD values calculated for backbone heavy atoms in $\AA$ in the 496 three parallel 5 ns long MD simulations with their standard deviations for all systems.

\begin{tabular}{ccccc} 
Chain & Structure & Neutral & $\mathbf{0 . 5} \mathbf{M}$ & $\mathbf{1 . 5} \mathbf{M}$ \\
\hline \hline A & Helices & $\mathbf{1 . 7 2} \pm 0.97$ & $\mathbf{1 . 7 4} \pm 1.20$ & $\mathbf{1 . 4 4} \pm 1.07$ \\
A & Turn & $\mathbf{1 . 5 6} \pm 0.24$ & $\mathbf{1 . 6 9} \pm 0.45$ & $\mathbf{1 . 2 8} \pm 0.28$ \\
A & Loop & $\mathbf{3 . 6 7} \pm 3.42$ & $\mathbf{2 . 9 8} \pm 1.77$ & $\mathbf{2 . 3 2} \pm 1.54$ \\
B & Helix & $\mathbf{1 . 8 7} \pm 0.87$ & $\mathbf{1 . 9 6} \pm 0.89$ & $\mathbf{1 . 6 6} \pm 0.79$ \\
B & Loops & $\mathbf{1 . 8 7} \pm 0.80$ & $\mathbf{2 . 0 0} \pm 0.79$ & $\mathbf{1 . 5 3} \pm 0.91$ \\
\hline
\end{tabular}

497

498

499

500 
Table 2. Number of water molecules in shells with their standard deviations around the protein averaged over all snapshots and three parallel MD simulations and total number of water molecules/ions in the simulation box as a function of salt concentrations

505

\begin{tabular}{cccc} 
Water layer & Neutral & $\mathbf{0 . 5} \mathbf{M}$ & $\mathbf{1 . 5} \mathbf{M}$ \\
\hline \hline $\mathbf{1}$ & $\mathbf{2 6 9} \pm 3$ & $\mathbf{2 6 9} \pm 4$ & $\mathbf{2 6 3} \pm 4$ \\
$\mathbf{1}_{\text {hydrophobic }}$ & $\mathbf{1 4 9} \pm 3$ & $\mathbf{1 5 0} \pm 3$ & $\mathbf{1 5 0} \pm 2$ \\
$\mathbf{1}_{\text {hydrophilic }}$ & $\mathbf{1 2 0} \pm 1$ & $\mathbf{1 1 9} \pm 3$ & $\mathbf{1 1 3} \pm 2$ \\
$\mathbf{2}$ & $\mathbf{2 9 7} \pm 4$ & $\mathbf{2 9 6} \pm 4$ & $\mathbf{2 8 7} \pm 3$ \\
$\mathbf{3}$ & $\mathbf{3 8 9} \pm 4$ & $\mathbf{3 8 6} \pm 5$ & $\mathbf{3 7 4} \pm 3$ \\
$\mathbf{4}$ & $\mathbf{4 9 3} \pm 5$ & $\mathbf{4 9 0} \pm 5$ & $\mathbf{4 7 8} \pm 4$ \\
$\mathbf{5}$ & $\mathbf{6 1 1} \pm 5$ & $\mathbf{6 0 7} \pm 4$ & $\mathbf{5 9 4} \pm 5$ \\
\hline $\mathbf{N}_{\text {water }}$ & $\mathbf{6 4 4 5}$ & $\mathbf{6 1 0 4}$ & $\mathbf{5 4 5 9}$ \\
$\mathbf{N}_{\text {sodium }}$ & $\mathbf{1}$ & $\mathbf{6 1}$ & $\mathbf{1 8 2}$ \\
$\mathbf{N}_{\text {chloride }}$ & $\mathbf{0}$ & $\mathbf{6 0}$ & $\mathbf{1 8 1}$ \\
\hline $\mathbf{N}_{\text {water, reference }}$ & $\mathbf{6 6 5 7}$ & $\mathbf{6 3 9 7}$ & $\mathbf{5 8 0 0}$
\end{tabular}

506 
Table 3. The average number of hydrogen bonds with their standard deviations formed between protein and $1^{\text {st }}$ solvation layer, and between the rest of the solvation shells at different salt concentrations. Data is averaged over the three parallel MD simulations and over all snapshots. "All" denotes the sum of all hydrogen bonds towards any partner. Solvation layers are designated by numbers.

\begin{tabular}{|c|c|c|c|}
\hline Hydrogen bond partners & Neutral & $0.5 \mathrm{M}$ & $1.5 \mathrm{M}$ \\
\hline $1^{\text {st }}-1^{\text {st }}$ & $1.84 \pm 0.01$ & $1.81 \pm 0.01$ & $1.76 \pm 0.00$ \\
\hline $1^{\text {st }}$ - protein & $\mathbf{0 . 5 6} \pm 0.02$ & $\mathbf{0 . 5 5} \pm 0.02$ & $\mathbf{0 . 5 5} \pm 0.01$ \\
\hline $1^{\text {st }}-$ protein $^{\text {hydrophilic }}$ & $1.25 \pm 0.04$ & $1.26 \pm 0.06$ & $1.26 \pm 0.03$ \\
\hline $1^{\text {st }}$ - all water & $\mathbf{3 . 0 5} \pm 0.02$ & $\mathbf{2 . 9 8} \pm 0.01$ & $\mathbf{2 . 8 4} \pm 0.03$ \\
\hline $1^{\text {st,hydrophilic }}$ - all water & $2.54 \pm 0.02$ & $\mathbf{2 . 4 9} \pm 0.02$ & $2.36 \pm 0.02$ \\
\hline $1^{\text {st,hydrophobic }}$ - all water & $3.46 \pm 0.01$ & $3.37 \pm 0.02$ & $\mathbf{3 . 1 8} \pm 0.02$ \\
\hline $1^{\text {st }}-$ all (protein+ water) & $\mathbf{3 . 6 1} \pm 0.02$ & $\mathbf{3 . 5 3} \pm 0.01$ & $\mathbf{3 . 3 9} \pm 0.02$ \\
\hline $2^{\text {nd }}-2^{\text {nd }}$ & $1.18 \pm 0.01$ & $1.14 \pm 0.01$ & $\mathbf{1 . 0 6} \pm 0.01$ \\
\hline $2^{\text {nd }}$ - all water & $2.91 \pm 0.01$ & $2.79 \pm 0.02$ & $2.57 \pm 0.02$ \\
\hline $3^{\text {rd }}-3^{\text {rd }}$ & $1.17 \pm 0.01$ & $1.13 \pm 0.01$ & $\mathbf{1 . 0 4} \pm 0.01$ \\
\hline $3^{\text {rd }}-$ all water & $3.69 \pm 0.01$ & $\mathbf{3 . 5 6} \pm 0.04$ & $\mathbf{3 . 2 8} \pm 0.03$ \\
\hline $4^{\text {th }}-4^{\text {th }}$ & $1.17 \pm 0.01$ & $1.13 \pm 0.01$ & $\mathbf{1 . 0 4} \pm 0.01$ \\
\hline $4^{\text {th }}-$ all water & $\mathbf{3 . 6 8} \pm 0.03$ & $\mathbf{3 . 5 4} \pm 0.05$ & $\mathbf{3 . 2 6} \pm 0.05$ \\
\hline reference systems & $\mathbf{3 . 4 0} \pm 0.01$ & $\mathbf{3 . 4 0} \pm 0.01$ & $\mathbf{3 . 1 4} \pm 0.01$ \\
\hline
\end{tabular}


515 Table 4. The average number of hydrogen bonds using various $\mathbf{C} \cdots \mathbf{O}$ distance criteria for 516 identifying hydrophobic water molecules in the first hydration shell in protein simulations at $0.5 \mathrm{M} \mathrm{NaCl}$ concentration. "All” denotes the sum of all hydrogen bonds towards any partner. Solvation layers are designated by numbers.

\begin{tabular}{ccccc} 
Hydrogen bond partners & $\mathbf{5 . 0 0} \AA$ & $\mathbf{4 . 5 0} \AA$ & $\mathbf{4 . 2 5} \AA$ & $\mathbf{4 . 0 0} \AA$ \\
\hline \hline $\mathbf{1}^{\text {st }}$ - all water & 3.07 & 2.99 & 2.93 & 2.85 \\
$\mathbf{2}^{\text {nd }}$ - all water & 2.82 & 2.80 & 2.79 & 2.77 \\
$\mathbf{3}^{\text {rd }}$ - all water & 3.58 & 3.58 & 3.58 & 3.58 \\
$\mathbf{4}^{\text {th }}-$ all water & 3.57 & 3.57 & 3.57 & 3.57
\end{tabular}


Table 5. The average number of hydrogen bonds at $0.5 \mathrm{M} \mathrm{NaCl}$ concentration calculated using various angle criteria for defining a hydrogen bond. "All" denotes the sum of all hydrogen bonds towards any partner. Solvation layers are designated by numbers.

\begin{tabular}{llll} 
Hydrogen bond partners & $\mathbf{1 2 0}^{\circ}$ & $\mathbf{1 3 0}^{\circ}$ & $\mathbf{1 4 5}^{\circ}$ \\
\hline \hline 1st $-\mathbf{1 s t}$ & 1.63 & 1.54 & 1.27 \\
$\mathbf{1 s t}-$ all water & 2.93 & 2.77 & 2.27 \\
$\mathbf{2}^{\text {nd }}-\mathbf{2}^{\text {nd }}$ & 1.14 & 1.07 & 0.85 \\
$\mathbf{2}^{\text {nd }}-$ all water & 2.54 & 2.38 & 1.92 \\
$\mathbf{3}^{\text {rd }}-\mathbf{3}^{\text {rd }}$ & 1.13 & 1.06 & 0.84 \\
$\mathbf{3}^{\text {rd }}-$ all water & 3.58 & 3.36 & 2.72 \\
$\mathbf{4}^{\text {th }}-\mathbf{4}^{\text {th }}$ & 1.14 & 1.06 & 0.85 \\
$\mathbf{4}^{\text {th }}-$ all water & 3.57 & 3.36 & 2.72
\end{tabular}


Table 6. The average energy $(\mathrm{kJ} / \mathrm{mol})$ of hydrogen bonds with their standard deviations layers.

\begin{tabular}{cccc} 
& Neutral & $\mathbf{0 . 5} \mathbf{M}$ & $\mathbf{1 . 5} \mathbf{M}$ \\
\hline \hline $\mathbf{1}^{\text {st }}-\mathbf{1}^{\text {st }}$ & $\mathbf{- 1 5 . 7 3} \pm 0.02$ & $\mathbf{- 1 5 . 6 8} \pm 0.01$ & $\mathbf{- 1 5 . 6 3} \pm 0.03$ \\
$\mathbf{1}^{\text {st,hydrophylic }}-\mathbf{1}^{\text {st,hydrophylic }}$ & $\mathbf{- 1 5 . 0 7} \pm 0.04$ & $\mathbf{- 1 4 . 9 6} \pm 0.03$ & $\mathbf{- 1 4 . 9 1} \pm 0.04$ \\
$\mathbf{1}^{\text {st,hydrophobic }-\mathbf{1}^{\text {st,hydrophobic }}}$ & $\mathbf{- 1 5 . 7 8} \pm 0.04$ & $\mathbf{- 1 5 . 7 0} \pm 0.04$ & $\mathbf{- 1 5 . 5 3} \pm 0.04$ \\
$\mathbf{1}^{\text {st }}-\mathbf{2}^{\text {nd }}$ & $\mathbf{- 1 5 . 1 4} \pm 0.02$ & $\mathbf{- 1 5 . 0 3} \pm 0.01$ & $\mathbf{- 1 4 . 8 3} \pm 0.03$ \\
$\mathbf{2}^{\text {nd }}-\mathbf{2}^{\text {nd }}$ & $\mathbf{- 1 4 . 9 5} \pm 0.01$ & $\mathbf{- 1 4 . 8 7} \pm 0.01$ & $\mathbf{- 1 4 . 6 8} \pm 0.02$ \\
$\mathbf{2}^{\text {nd }}-\mathbf{3}^{\text {rd }}$ & $\mathbf{- 1 5 . 4 9} \pm 0.01$ & $\mathbf{- 1 5 . 3 4} \pm 0.01$ & $\mathbf{- 1 5 . 0 5} \pm 0.03$ \\
$\mathbf{3}^{\text {rd }} \mathbf{3}^{\text {rd }}$ & $\mathbf{- 1 5 . 0 1} \pm 0.02$ & $\mathbf{- 1 4 . 8 8} \pm 0.01$ & $\mathbf{- 1 4 . 6 5} \pm 0.01$ \\
$\mathbf{3}^{\text {rd }}-\mathbf{4}^{\text {th }}$ & $\mathbf{- 1 5 . 5 0} \pm 0.01$ & $\mathbf{- 1 5 . 3 4} \pm 0.01$ & $\mathbf{- 1 5 . 0 3} \pm 0.02$ \\
$\mathbf{4}^{\text {th }}-\mathbf{4}^{\text {th }}$ & $\mathbf{- 1 5 . 2 0} \pm 0.02$ & $\mathbf{- 1 4 . 9 8} \pm 0.01$ & $\mathbf{- 1 4 . 6 5} \pm 0.01$ \\
$\mathbf{4}^{\text {th }}-\mathbf{5}^{\text {th }}$ & $\mathbf{- 1 5 . 5 1} \pm 0.01$ & $\mathbf{- 1 5 . 3 5} \pm 0.01$ & $\mathbf{- 1 5 . 0 1} \pm 0.01$ \\
\hline Reference systems & $\mathbf{- 1 5 . 9 1} \pm 0.01$ & $\mathbf{- 1 5 . 6 7} \pm 0.01$ & $\mathbf{- 1 5 . 5 5} \pm 0.01$
\end{tabular}



calculated using various angle criteria for defining a hydrogen bond. Solvation layers are designated by numbers.

\begin{tabular}{cccc} 
& $\mathbf{1 2 0}^{\circ}$ & $\mathbf{1 3 0}^{\circ}$ & $\mathbf{1 4 5}^{\circ}$ \\
\hline \hline $\mathbf{1}^{\text {st }}-\mathbf{1}^{\text {st }}$ & -15.66 & -16.79 & -18.21 \\
$\mathbf{1}^{\text {st }}-\mathbf{2}^{\text {nd }}$ & -15.11 & -16.04 & -17.42 \\
$\mathbf{2}^{\text {nd }}-\mathbf{2}^{\text {nd }}$ & -14.86 & -16.08 & -17.58 \\
$\mathbf{2}^{\text {nd }}-\mathbf{3}^{\text {rd }}$ & -15.32 & -16.20 & -17.54 \\
$\mathbf{3}^{\text {rd }}-\mathbf{3}^{\text {rd }}$ & -14.86 & -16.12 & -17.58 \\
$\mathbf{3}^{\text {rd }}-\mathbf{4}^{\text {th }}$ & -15.32 & -16.20 & -17.50 \\
$\mathbf{4}^{\text {th }}-\mathbf{4}^{\text {th }}$ & -15.16 & -16.08 & -17.33 \\
$\mathbf{4}^{\text {th }}-\mathbf{5}^{\text {th }}$ & -15.37 & -16.08 & -17.25 \\
$\mathbf{5}^{\text {th }}-\mathbf{5}^{\text {th }}$ & -14.91 & -15.66 & -16.87
\end{tabular}

534

535 


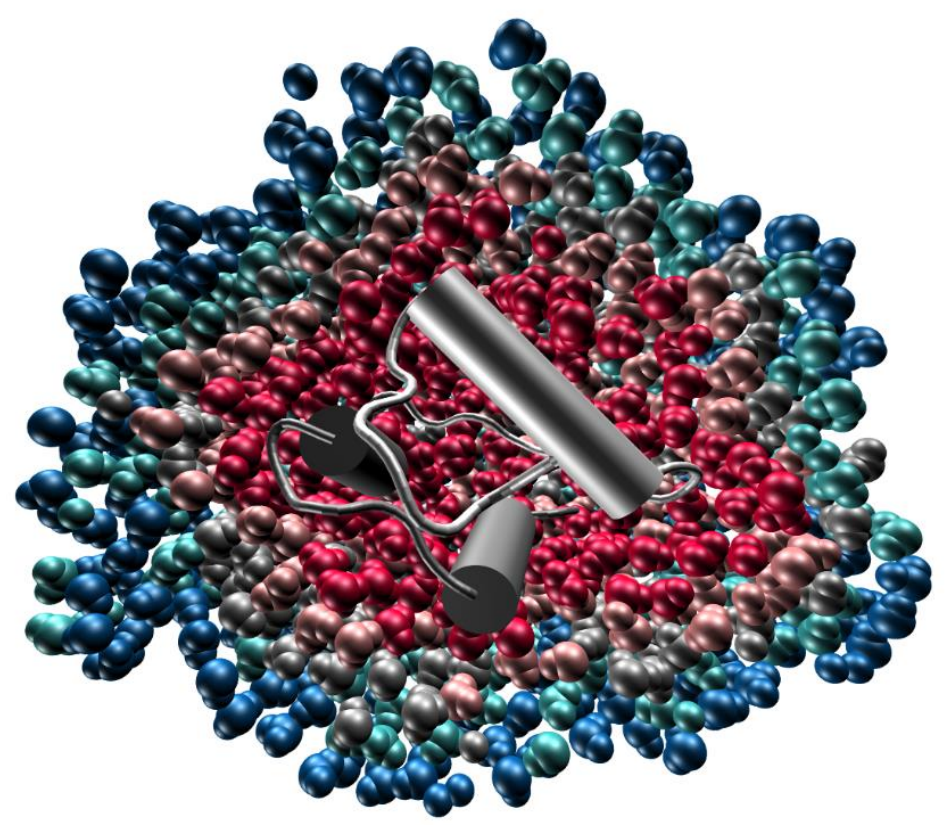

536

537

Figure 1. Schematic representation of the hydration sphere layers around the protein. 


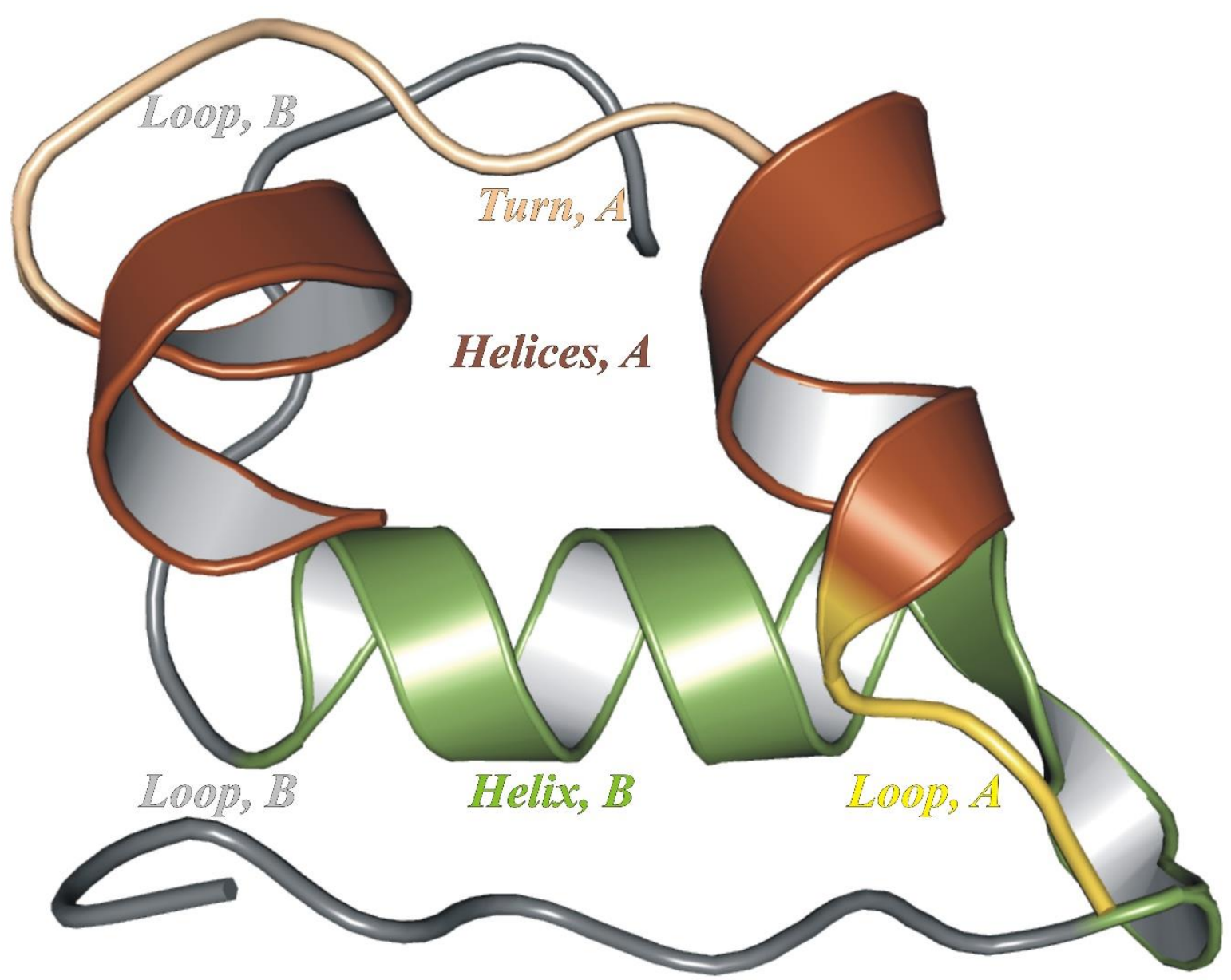

Figure 2. Structural elements in the insulin monomer. Colors used to depict the various structural elements in chain A : brown - helices; wheat - turn; yellow - loop; while in chain B: 


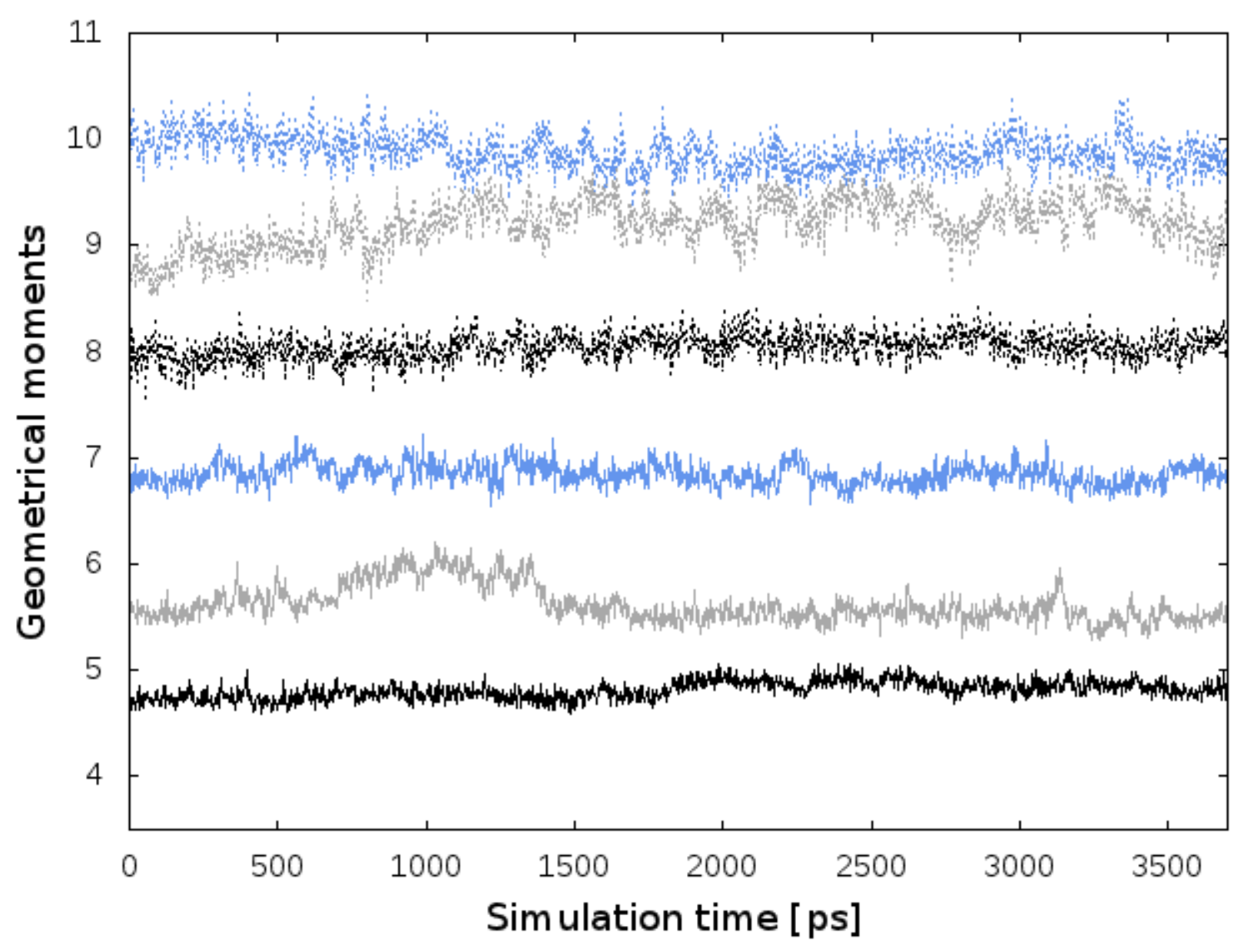

Figure 3. The inertia moments during the simulations. Inercia momements belong to protein shown with lines. Data include the protein and the first three water layers are represented with dotted lines. The three moment components are shown in black, gray and ice blue. 


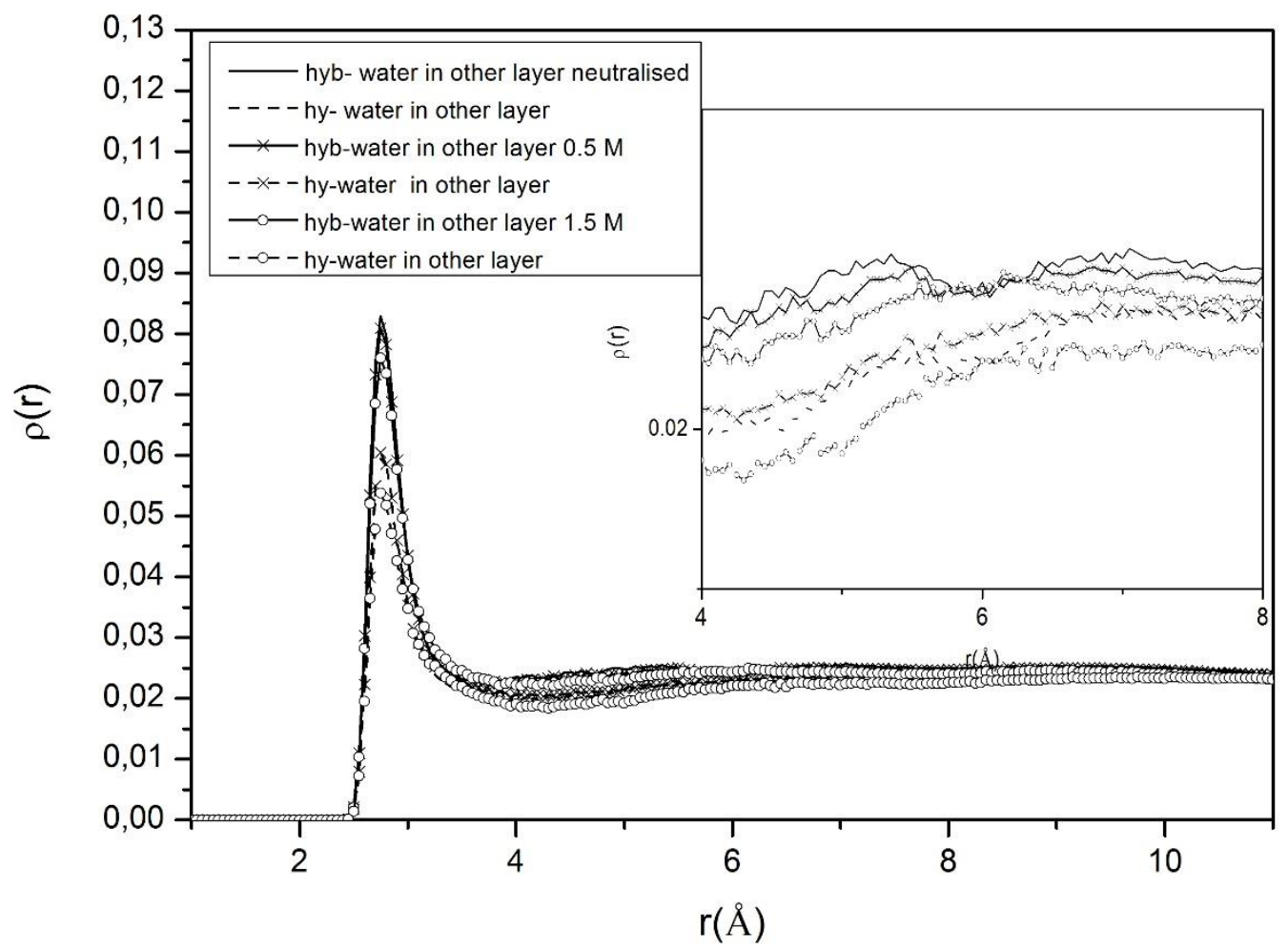

Figure 4. Decomposition of the raw density distribution function (of-the $\mathbf{O}_{\text {water- }}-\mathrm{O}_{\text {water }}$ distance) of water molecules in the first hydration layer interacting with water molecules in outer layers according to the hydrophilic (it forms H-bonds with the protein) or hydrophobic 


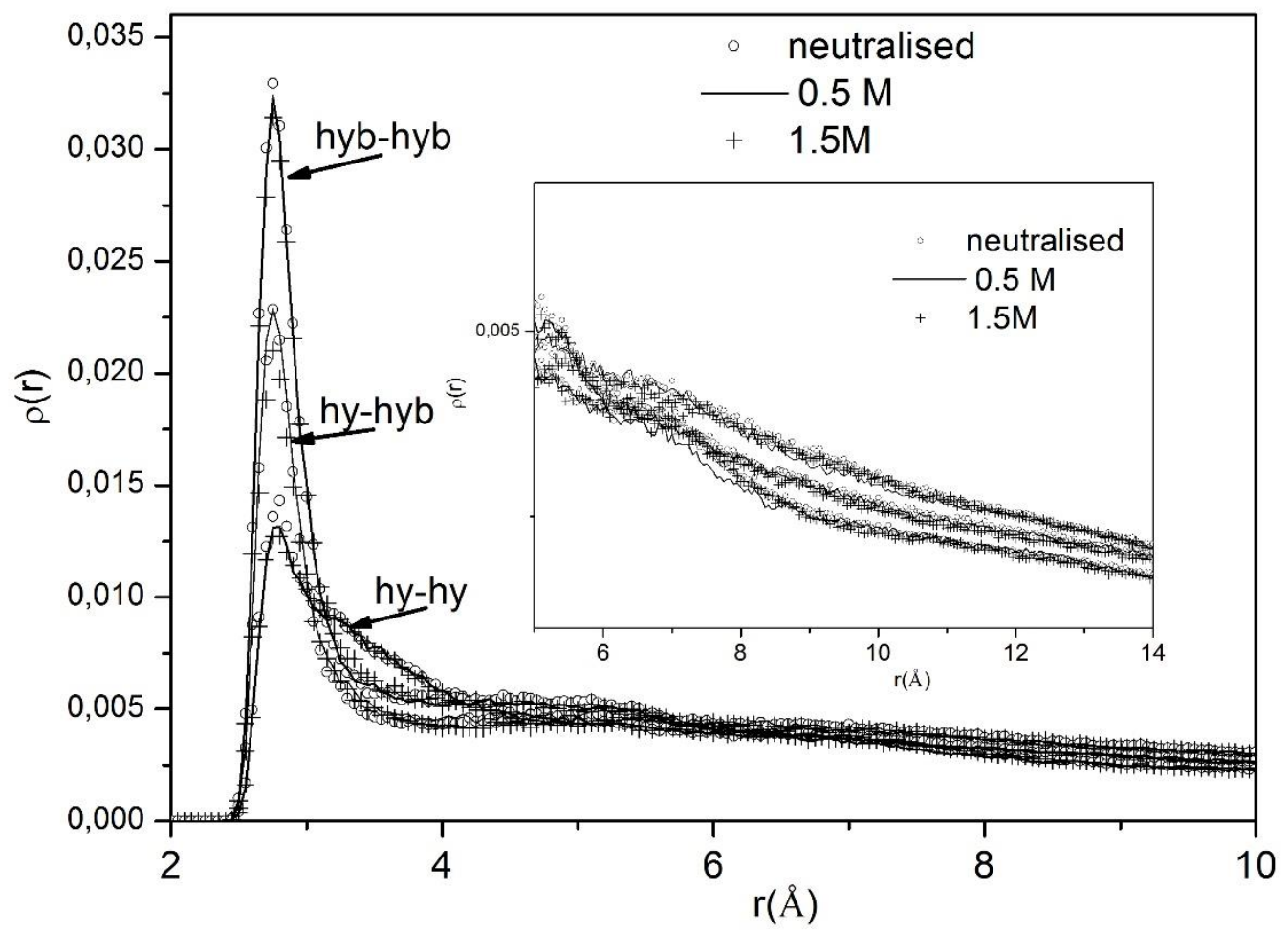

558 Figure 5 Decomposition of the raw density distribution function (of-the $\mathbf{O}_{\text {water- }} \mathbf{O}_{\text {water }}$ distance) among water molecule in the first hydration shell according to the nature of interacting water molecules: Hydrophobic water: it forms no H-bonds with the protein, hydrophylic water: it forms $\mathbf{H}$-bonds with the protein at various salt concentrations. 